\title{
Polite patients receive better care but "incivility in clinics and hospitals is rampant"
}

\author{
E Cite as: CMAJ 2019 April 23;191:E457. doi: 10.1503/cmaj.109-5741
}

Posted on cmajnews.com on Apr. 3, 2019.

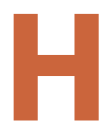

ow patients and their families treat doctors may affect the quality of care they receive. There's growing evidence that exposure to rudeness undermines physician performance, and a recent study in Pediatrics shows the reverse may also be true.

Researchers at Tel Aviv University in Israel found that neonatal intensive care unit teams performed better in training simulations when they had positive interactions with a patient or patient's family.

"Incivility in clinics and hospitals is rampant," according to coauthor Peter Bamberger. "If patients and families of patients treat medical staff with a degree of respect and kindness, they are likely to receive better care. It's that simple."

The researchers observed 43 teams during a training workshop. Some teams received gratitude from the mother of an infant patient, while others received praise from a physician-expert, from both a mother and a physicianexpert, or from neither. Positive feedback from patients' families enhanced performance, largely because it boosted team communication.

"Professional feedback had little effect," Bamberger noted.

Another recent study he coauthored found that gratitude from colleagues can actually be harmful in certain situations. The study in Human Relations observed surgical teams over six months to see how often staff deviated from protocol after receiving praise or criticism from colleagues. According to Bamberger, "positive interactions in the course of a complex task like surgery did not yield a positive effect." In some cases, it had an adverse effect on performance. "Perhaps if you're

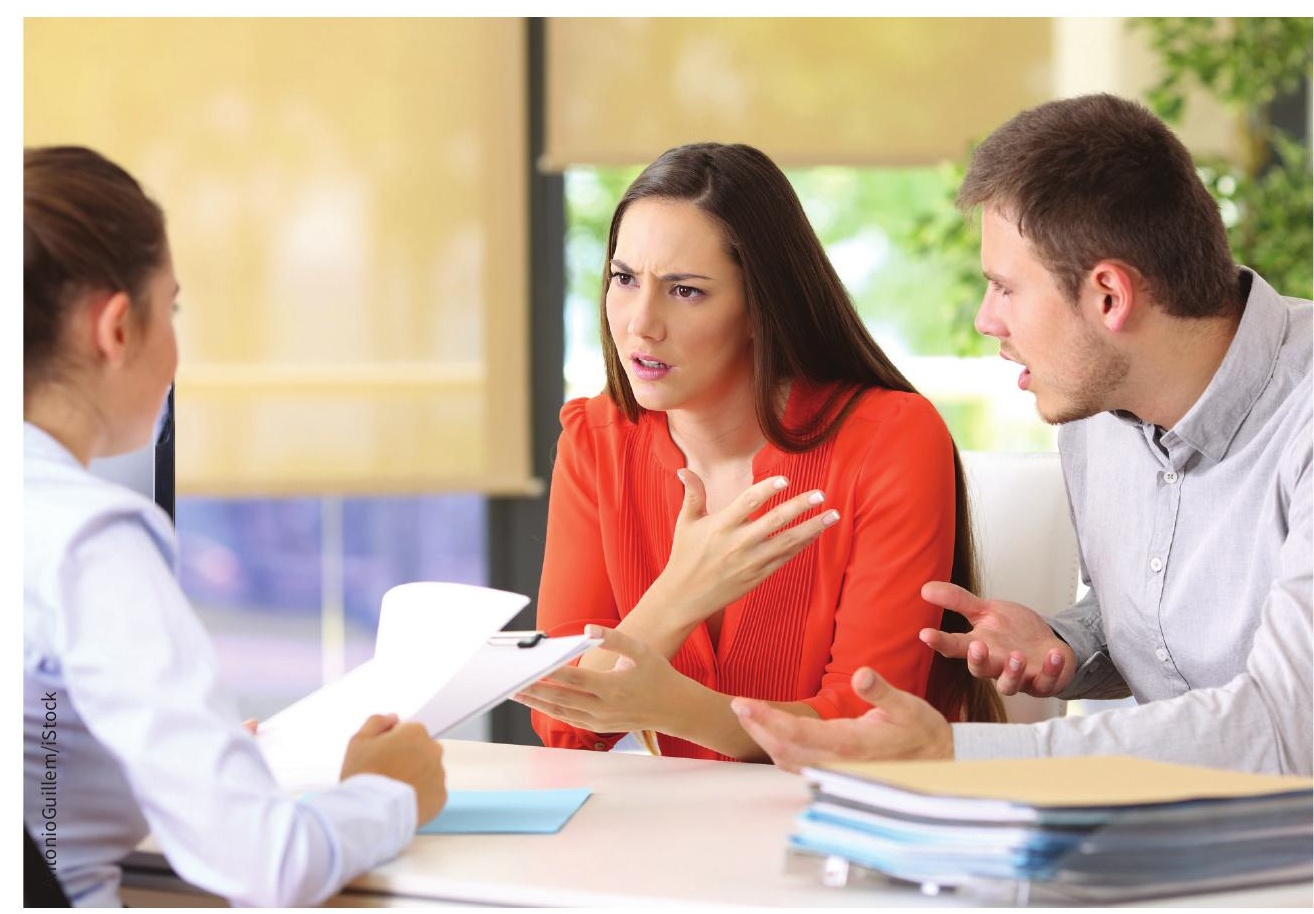

Doctors may think they can rise above rudeness from patients to deliver top-notch care, but recent studies suggest otherwise.

pleased, you become complacent or overconfident and neglect to pick up on critical signals."

Meanwhile, there's growing evidence of the dangers of rudeness. A study published this month linked incidents of rudeness in health care (mostly from patients and families) to reduced compliance by health workers with infection control and medication protocols. Bamberger and colleagues' Pediatrics study builds on research published in 2017 that found neonatal intensive care unit teams exposed to rude comments from a patient's mother performed worse across diagnostic, intervention and teamwork measures. An earlier study found similar negative effects on individual per- formance when a physician-expert made rude remarks.

According to Amir Erez of the University of Florida, a coauthor of all three papers, "even if doctors have the best intentions in mind, as they usually do, they cannot get over rudeness because it interferes with their cognitive functioning without an ability to control it."

These studies indicate that seeking help, sharing information and cognitive bias modification tools mediate the effects of rudeness. But according to Bamberger, "the bottom line here is that, as a patient, you should be nice and express gratitude to medical practitioners."

Lauren Vogel, CMAJ 